\title{
Correction: Wilson, N.G., et al. Tropical Range Extension for the Temperate, Endemic South-Eastern Australian Nudibranch Goniobranchus splendidus (Angas, 1864). Diversity 2016, 8, 16
}

\author{
Nerida G. Wilson ${ }^{1,2, *(1)}$, Anne E. Winters ${ }^{3}$ and Karen L. Cheney ${ }^{3}$ \\ 1 Western Australian Museum, 49 Kew Street, Welshpool, WA 6106, Australia \\ 2 School of Animal Biology, University of Western Australia, Crawley, WA 6009, Australia \\ 3 School of Biological Sciences, The University of Queensland, St Lucia, QLD 4072, Australia; \\ e.anne06@gmail.com (A.E.W.); k.cheney@uq.edu.au (K.L.C.) \\ * Correspondence: nerida.wilson@museum.wa.gov.au; Tel.: +61-08-9212-3844
}

Received: 5 July 2018; Accepted: 5 July 2018; Published: 5 July 2018

check for updates

The authors wish to make the following corrections to this paper [1]. The details are as follows:

1. In Table 1, the pending Genbank numbers should be MH018011, MH017998, MH018010, MH01797. Please, replace Table 1

Table 1. Species of Goniobranchus included in the phylogenetic analysis.

\begin{tabular}{ccccc}
\hline Species & Voucher & Locality & COI & 16 S \\
\hline G. albopustulosus & CASIZ 142953 & Maui, Hawaii & JQ 727828 & JQ 727701 \\
G. daphne & UQ collection & Gold Coast Seaway, QLD & KJ 001297 & KJ 018921 \\
G. epicurea & SAM D 19285 & Tasmania, Australia & EF 535114 & AY 458804 \\
G. heatherae & CASIZ 175546 & Cape Peninsula, South Africa & JQ 727844 & JQ 727720 \\
G. kuniei & SAM D 19261 & Heron Is., GBR, QLD & EF 535112 & AY 458807 \\
G. splendidus & SAM D 19292 & Mooloolaba, QLD & EF 535115 & AY 458815 \\
G. splendidus & CASIZ 146039 & Mooloolaba, QLD & EU 982738 & EU 982789 \\
G. cf. splendidus & UQ 1102 & Mackay, QLD & pending & pending \\
G. tasmaniensis & SAM 19295 & Triabunna, Tasmania & EF 535113 & AY 458817 \\
G. tinctorius & UQ 1097 & Mackay, QLD & pending & pending \\
G. verrieri & CASIZ 158796 & Batangas, Philippines & JQ 727858 & JQ 727740 \\
\hline
\end{tabular}

with the following

Table 1. Species of Goniobranchus included in the phylogenetic analysis.

\begin{tabular}{ccccc}
\hline Species & Voucher & Locality & COI & 16 S \\
\hline G. albopustulosus & CASIZ 142953 & Maui, Hawaii & JQ 727828 & JQ 727701 \\
G. daphne & UQ collection & Gold Coast Seaway, QLD & KJ 001297 & KJ 018921 \\
G. epicurea & SAM D 19285 & Tasmania, Australia & EF 535114 & AY 458804 \\
G. heatherae & CASIZ 175546 & Cape Peninsula, South Africa & JQ 727844 & JQ 727720 \\
G. kuniei & SAM D 19261 & Heron Is., GBR, QLD & EF 535112 & AY 458807 \\
G. splendidus & SAM D 19292 & Mooloolaba, QLD & EF 535115 & AY 458815 \\
G. splendidus & CASIZ 146039 & Mooloolaba, QLD & EU 982738 & EU 982789 \\
G. cf. splendidus & UQ 1102 & Mackay, QLD & MH018011 & MH017998 \\
G. tasmaniensis & SAM 19295 & Triabunna, Tasmania & EF 535113 & AY 458817 \\
G. tinctorius & UQ 1097 & Mackay, QLD & MH018010 & MH01797 \\
G. verrieri & CASIZ 158796 & Batangas, Philippines & JQ 727858 & JQ 727740 \\
\hline
\end{tabular}


2. Please note that Figure 2 contains a mistake, splendidus Mackay (UQ1097) should be splendidus Mackay (UQ1102). Please replace Figure 2

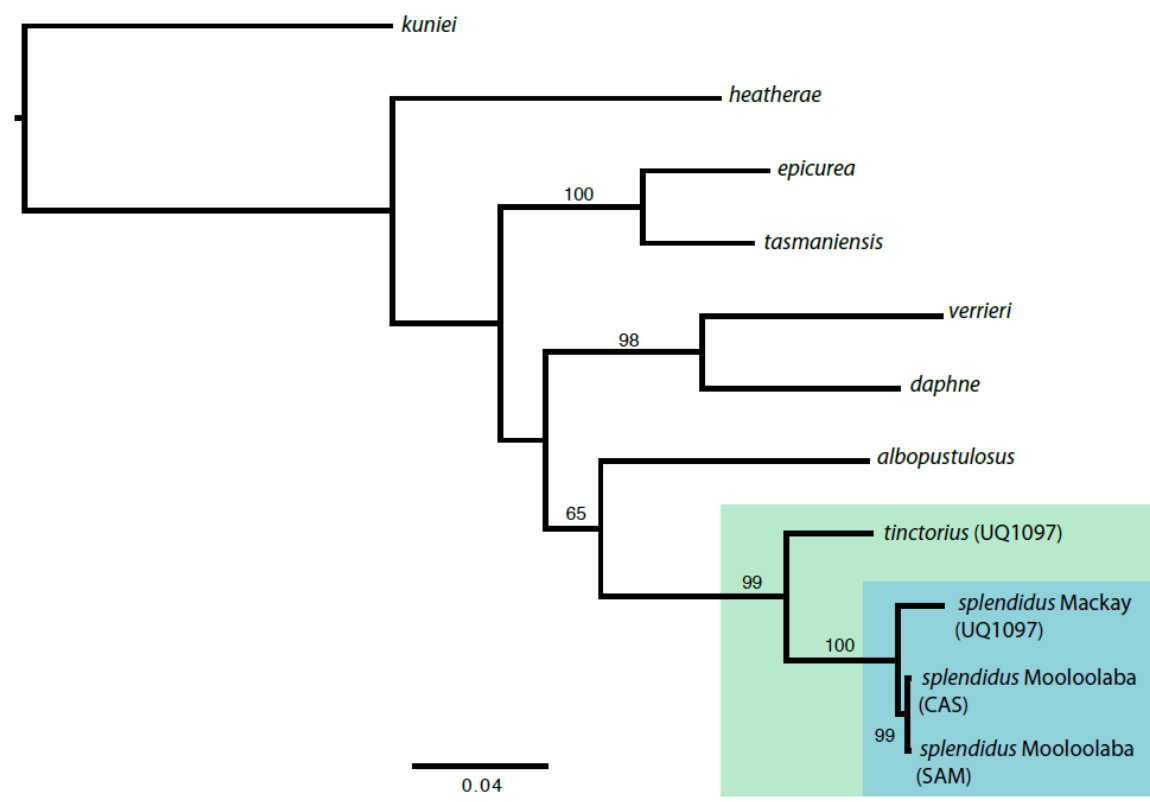

Figure 2. Maximum-likelihood topology for Cytochrome Oxidase I gene (COI) + 16S DNA from selected species of Goniobranchus nudibranchs. Specimens of G. splendidus shown in blue box, and sister species G. tinctorius shown in green box. Bootstrap values less than 60 are not shown.

with the following

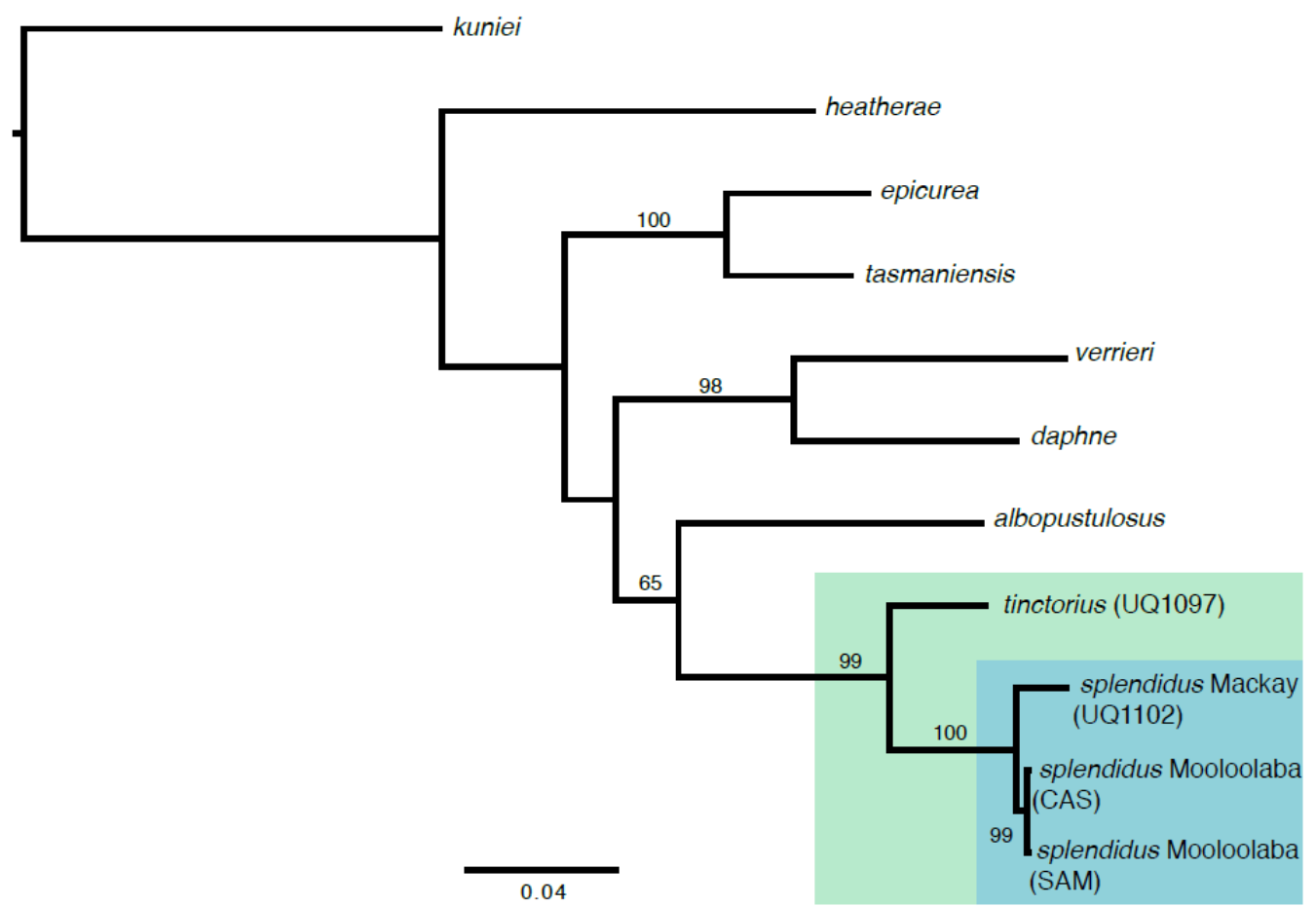

Figure 2. Maximum-likelihood topology for Cytochrome Oxidase I gene (COI) + 16S DNA from selected species of Goniobranchus nudibranchs. Specimens of G. splendidus shown in blue box, and sister species G. tinctorius shown in green box. Bootstrap values less than 60 are not shown. 
We apologize for any inconvenience caused to the readers by these changes. The manuscript will be updated, and the original publication will remain available from the article webpage.

\section{Reference}

1. Wilson, N.G.; Winters, A.E.; Cheney, K.L. Tropical Range Extension for the Temperate, Endemic South-Eastern Australian Nudibranch Goniobranchus splendidus (Angas, 1864). Diversity 2016, 8, 16. [CrossRef] 